stability domains. Our objective was to develop a conceptually novel performance-based assessment to quantify balance ability of CLOAs that minimizes current limitations and decreases test time by using a non-fixed-item format that eliminates completing all items to obtain a score.

This methodological study consisted of 3 phases. In phase 1, we developed a 26-item, dichotomously-scored preliminary Performance-based Balance Scale (PBS) by (1) using an expert panel to build the scale by adapting items from existing balance measures and organizing items by difficulty into posture stability domains, (2) pilot testing, and (3) post-test revision. Phase 2 involved testing the preliminary PBS with 35 subjects (10 men, 25 women, mean age $=75.9,60-91$ yrs). Using Rasch dichotomous model analysis, we created a non-fixed-item PBS format and identified 2 items to serve as starting points for testing based on performance ability; thereby, eliminating the need to test all items. In phase 3, we re-tested the same subjects 4-8 weeks later. Spearman's rho was used to correlate the preliminary and non-fixeditem PBS to determine validity of the non-fixed-item format resulting in $r s=0.94$.

In conclusion, the PBS is a novel, 20-item, non-fixed format clinical balance test with 2 entry points for CLOAs that minimizes ceiling effects, assesses different domains, and reduces test time.

\section{INBED: INEXPENSIVE NODE FOR BED-EXIT DETECTION: A WEARABLE FALL PREVENTION SYSTEM}

N. Jaehne-Raden ${ }^{1}$, U. Kulau ${ }^{2}$, K. Wolf ${ }^{1}$, H. Meyer zu Schwabedissen ${ }^{3}$, M. Marschollek ${ }^{1}, 1$. Peter L. Reichertz Institute for Medical Informatics University of Braunschweig - Institute of Technology and Hannover Medical School, Hanover, Lower Saxony, Germany, 2. Institute of Operating Systems and Computer Networks, Braunschweig, Lower Saxony, Germany, 3. Clinic for rheumatism and diseases of the elderly at the Municipal Hospital Braunschweig, Braunschweig, Lower Saxony, Germany

Age-related falls are a widespreaded and expensive issue of society and with this a big challenge of gerontological research. Hence we present INBED - an inexpensive, modular and body-worn (medio-femoral) fall prevention system. Compared to existing solutions, INBED offers a more proper solution to prevent falls in clinical and elderly care environments.

The INBED detects several events such as getting up from a sitting or lying position. These events are broadcasted wirelessly to notify the nursing stuff, so that they can assist the respective care recipient accordingly. In further consequence, highly critical fall events can be avoided due to the early alert of the nursing staff. The INBED can replace the commonly used belt or bed rail systems for high risk fall patients. Thus, the personal range of movement and the self-determination of the patient will be supported without reducing the sense of protection. INBED itself is a approx. $2 \times 2 \mathrm{~cm}$ wearable device that contains several sensors, a microcontroller unit and a wireless radio transceiver.

Development objectives were:

- development of a reliable and cost-effective solution for a sensor-based uprise-detection for fall prevention established by first research results with different sensor prototypes.
- apart from the function of immediate uprise-detection, the system can also recognize states of restlessness occurring in advance of patient's rising, so that the caregiver can intervene before the first uprise.

- in addition to above-mentioned functions, the system can be used to implement a virtual fence. Patients can be protected from risk areas, e.g. stairs, by sending a dedicated event.

\section{SESSION 4120 (SYMPOSIUM)}

\section{THE INTERSECTION OF TRAUMA AND DISASTER BEHAVIORAL HEALTH}

Chair: K.E. Cherry, Louisiana State University, Baton

Rouge, Louisiana

Discussant: A. Gibson, Winthrop University, Rock Hill, South Carolina

Disasters bring catastrophic destruction with loss of life, homes, and property. The disaster science literature documents threats to health and well-being for directly affected individuals during the immediate impact period, although fewer studies have addressed psychological health among disaster survivors and indirectly affected disaster volunteers who assist with response and recovery in the years after a destructive environmental event. In this symposium, the intersection of trauma and disaster behavioral health will be examined from a lifespan perspective. Five papers will be presented that include behavioral data collected with samples of older adults exposed to four different environmental events: the 2015 South Carolina floods; the 2005 Atlantic Hurricanes Katrina and Rita, the 2007 wildfires in San Diego County, CA, and the 2010 BP Deepwater Horizon Oil Spill. Risk and resilience factors among survivors and disaster relief volunteers will be highlighted based on qualitative analyses of narrative text and quantitative analyses of survey and questionnaire data. Taken together, these five papers will provide new evidence concerning psychological and social factors that may lessen vulnerability and promote resilience for directly and indirectly affected older adults in the years after a natural disaster. Lessons learned from these prior disasters offer new insights into evacuation safety and the response and recovery phases of a disaster with an aging population. Implications for disaster preparedness and behavioral health and policy recommendations will be discussed.

\section{AN INQUIRY INTO OLDER DISASTER RESPONDERS' SECONDARY TRAUMATIC STRESS}

A. Gibson, University of Kentucky's College of Social Work, Lexington, Kentucky

Secondary Traumatic Stress (STS) symptoms are common among disaster responders. An exploratory study, using primarily qualitative methods was conducted to gain a better understanding STS in disaster response volunteers 60 and older. Fifty-eight older disaster responders involved with the 2015 South Carolina floods participated in this research effort. As part of this study, questions regarding STS were explored. STS levels among volunteer responders who provided care to victims of varying disasters varied substantially, but common themes emerged that represent both risk and protective factors for STS. Previous exposure 